\title{
Relationship of aortic cross-sectional area to height ratio and the risk of aortic dissection in patients with bicuspid aortic valves
}

\author{
Lars G. Svensson, MD, PhD, ${ }^{a, b}$ Kyung-Hwan Kim, MD, ${ }^{a, b}$ Bruce W. Lytle, MD, and Delos M. Cosgrove, MD, ${ }^{a, b}$ \\ Cleveland, Ohio
}

T

he relationship between the size of the aorta, in particular the cross-sectional area, and the patient's height expressed as a ratio and the risk of aortic dissection has not been determined in patients with bicuspid aortic valves.

\section{Methods and Patients}

Between January 4, 1993, and September 9, 2002, 430 patients underwent surgical intervention for bicuspid aortic valves and ascending aorta with or without aortic arch repair by the authors at either the Lahey Clinic or The Cleveland Clinic Foundation. Forty patients had aortic dissection, and 25 dissections were acute. Thirty-six patients were male, and 4 were female. The mean age was 49.8 years \pm 13.4 years (range, $17-80$ years). Six patients had grade 0 aortic valve regurgitation, 5 had $1+, 10$ had $2+, 7$ had $3+$, 2 had $4+$, and 7 had aortic valve stenosis. Ten patients had separate aortic valve replacements, 26 had composite valve grafts, and 3 underwent aortic valve repair and root remodeling or reimplantation operations.

Hospital charts, including results of computed tomographic, magnetic resonance imaging, and echocardiographic studies, were reviewed to determine the size of the aorta recorded before aortic replacement. In addition, the maximal cross-sectional area in square centimeters was obtained. This was divided by the patient's height in meters so that a ratio could be determined as follows:

$$
\text { Ratio }=\mathrm{r}^{2} \pi\left(\mathrm{cm}^{2}\right) / \text { height }(\mathrm{m}) .
$$

\section{Results}

Thirty-eight (95\%) of the 40 patients were 30-day in-hospital survivors. Four $(10 \%)$ patients had a stroke, and 2 strokes were permanent. For the 390 nondissection patients, 385 (98.7\%) were 30-day survivors.

Table 1 shows aortic diameters. Of interest, 5 (12.5\%) patients had an aortic diameter of less than $5 \mathrm{~cm}$. The mean diameter of the

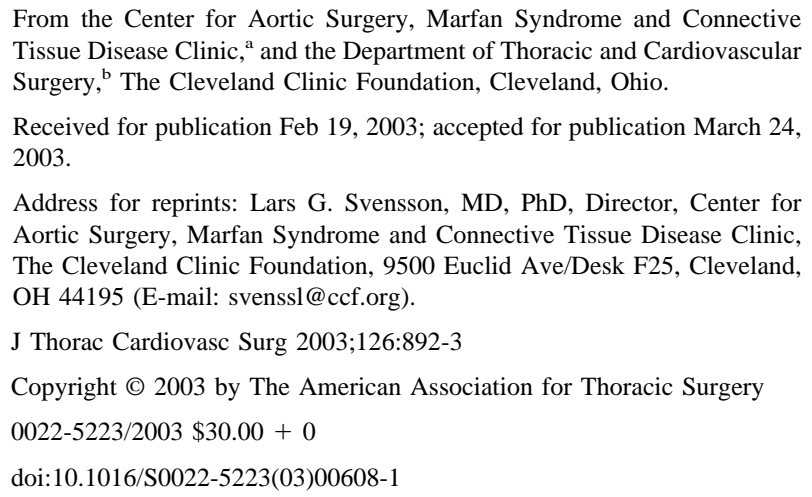

TABLE 1. Aortic diameters measured before surgical intervention

\begin{tabular}{lrl}
\hline Diameter $(\mathbf{c m})$ & N & $\%$ \\
\hline$<5$ & 5 & 12.5 \\
$5-5.5$ & 9 & 22.5 \\
$5.6-6$ & 12 & 30 \\
$6.1-7$ & 8 & 20 \\
$>7$ & 6 & 15
\end{tabular}

Clearly, measuring the size of the aorta immediately before the operation would overestimate the size at which aortic dissection occurred because it is likely that the aorta enlarged in most patients once the dissection occurred. Thus the true size at which aortic dissection occurs might be smaller.

aorta was $6.02 \pm 1.47 \mathrm{~cm}$ (range, $3-10.8 \mathrm{~cm}$ ). The mean ratio was $18.34 \pm 8.88$ (range, 7-54). Figure 1 shows the relationship of aortic cross-sectional size to height. Note that dissection occurred at a smaller diameter for shorter patients. As expected from the formula, the risk of dissection was constant for any given height according to the ratio (Figure 2).

\section{Discussion}

The reason for operating on asymptomatic patients with dilated aortas is to prevent rupture or aortic dissection. Most surgeons recommend operations when the aortic diameter exceeds $5.5 \mathrm{~cm}$ in size. Of note (Table 1), $35 \%$ of patients had dissections at $5.5 \mathrm{~cm}$ or less. Studies have shown that the average size at which dissection or rupture occurs in the ascending aorta is $5.9 \mathrm{~cm} .{ }^{2}$ In patients with Marfan syndrome, recommendations for repair of the aorta have included $5 \mathrm{~cm}, 5.5 \mathrm{~cm}, 1.5$ times the normal-sized aortic arch, or a cross-sectional area/height ratio exceeding $10 .^{1,3,4}$

The empiric use of 1.5 or even 2.0 times the aortic arch size has been shown for patients with Marfan syndrome in a prospective prophylactic $\beta$-blocker study to be inadequate in preventing aortic dissection. ${ }^{1}$ Furthermore, the present use of axial computed tomography or magnetic resonance imaging studies rather than anterior oblique angiograms makes determination of the midaortic arch size difficult to quantify.

On the basis of this study, it is clear that aortic dissection can occur at a smaller size than generally perceived. Fifteen percent of patients with Marfan syndrome have aortic dissection at a size of less than $5 \mathrm{~cm} .{ }^{1,3}$ In this study the number is similar (12.5\%). Both in patients with bicuspid valve-related aneurysms and patients with the Marfan syndrome, loss of elastic tissue in the aortic wall is a hallmark. These data would suggest that the risk of aortic dissection in patients with bicuspid valves, according to size or ratio, is not dissimilar from that in patients with the Marfan syndrome. ${ }^{1,3,4}$ The correlation of normal aortic size to height has 


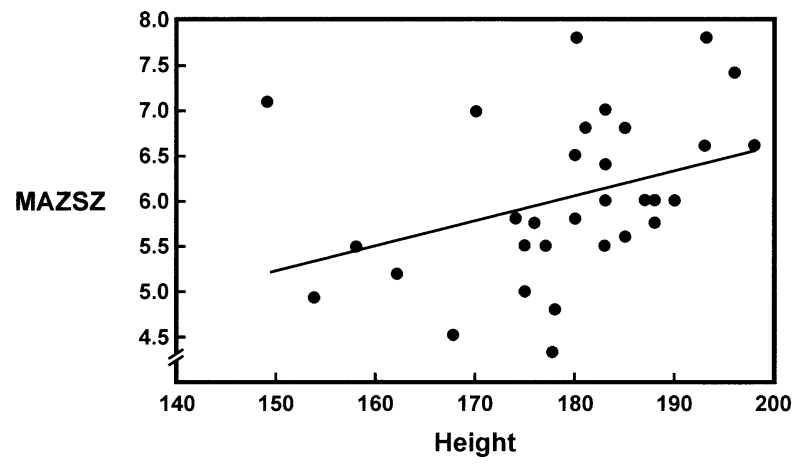

Figure 1. Relationship between aortic cross-sectional diameter and height at the size at which the aortic dissection was noted. MAZSZ, Maximum aortic size.

previously been documented in the Framingham study. ${ }^{5}$ Indexing the cross-sectional area of aneurysms to the patient's height would take into account the likely greater risk of dissection for the equivalent size in short patients when compared with tall patients, as shown in Figure 1. ${ }^{1}$ Use of the cross-sectional area also takes into account the likely exponential risk of dissection or rupture related to increasing aortic size because the radius squared is used in the calculation rather than the linear variable of diameter. ${ }^{1}$ Figure 2 confirms that the risk is constant according to height if the ratio is calculated. Furthermore, as in patients with the Marfan syndrome, ${ }^{1}$ if patients had undergone surgical intervention for a ratio of greater than $10,95 \%$ of dissections would theoretically have been prevented. Indeed, with a mean ratio of 18.34 and subtraction of 1 SD (8.88), the ratio would be 9.46 .

Because of the low operative risk for elective bicuspid valve repair or replacement and aortic surgery, (98.7\% in our experience), we would recommend more aggressive treatment for

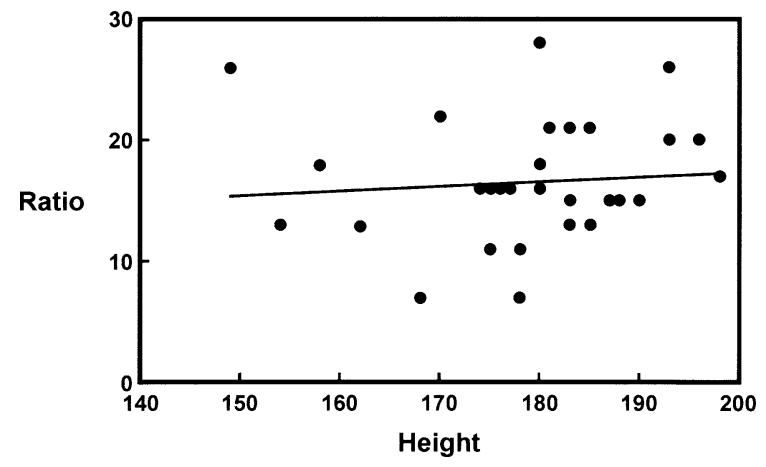

Figure 2. Relationship between ratio and height.

asymptomatic aortic aneurysms and that an aorta of greater than $4.5 \mathrm{~cm}$ or a ratio of greater than 10 be repaired for symptomatic bicuspid aortic valve disease.

\section{References}

1. Svensson LG, Khitin L. Aortic cross-sectional area/height ratio timing of aortic surgery in asymptomatic patients with Marfan syndrome. J Thorac Cardiovasc Surg. 2002;123:360-1.

2. Coady MA, Rizzo JA, Hammond GL, Kopf GS, Elefteriades JA. Surgical intervention criteria for thoracic aortic aneurysms: a study of growth rates and complications. Ann Thorac Surg. 1999;67:1922-6.

3. Gott VL, Greene PS, Alejo DE, Cameron DE, Naftel DC, Miller DC, et al. Replacement of the aortic root in patients with Marfan's syndrome. $N$ Engl J Med. 1999;340:1307-13.

4. Smith JA, Fann JI, Miller DC, Moore KA, DeAnda A, Mitchell RS, et al. Surgical measurement of aortic dissection in patients with Marfan syndrome. Circulation. 1994;90:235-42.

5. Vasan RS, Larson MG, Levy D. Determinants of echocardiographic aortic root size: the Framingham Heart Study. Circulation. 1995;91: 734-40. 